ARTÍCULO ORIGINAL

\title{
USO DE ANTIBIÓTICOS EN PACIENTES INTERNADOS EN UN HOSPITAL NACIONAL DE LIMA, PERÚ
}

\author{
Cristhian Resurrección-Delgado(iD)1,2,a,b , Alfredo Chiappe-Gonzalez(iD)1,a,a, , José Bolarte-

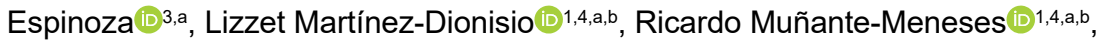 \\ Yuriko Vicente-Lozano (1D)1,4,a,b, Paola Rondan-Guerrero (D1,4,a, \\ Wendy Chávarry-Velásquez (i) 1,4,a , Jaime Álvarezcano-Berroa (i] ${ }^{3, a, b}$, \\ Juan Montenegro-Idrogo (iD) ${ }^{1,5, a, b}$ \\ ${ }^{1}$ Servicio de Enfermedades Infecciosas y Tropicales, Hospital Nacional Dos de Mayo, Lima, Perú. \\ ${ }^{2}$ Universidad Peruana de Ciencias Aplicadas (UPC), Lima, Perú. \\ ${ }^{3}$ Departamento de Epidemiología, Hospital Nacional Dos de Mayo, Lima, Perú. \\ ${ }^{4}$ Universidad Nacional Mayor de San Marcos, Lima, Perú. \\ ${ }^{5}$ Facultad de Ciencias de la Salud, Universidad Científica del Sur, Lima, Perú.

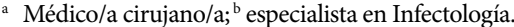

\section{RESUMEN}

Objetivo: Determinar la prevalencia puntual y características del uso de antibióticos en distintas salas de hospitalización del Hospital Nacional Dos de Mayo. Materiales y métodos: Se realizó un estudio transversal de tres semanas de duración mediante un abordaje censal de los pacientes hospitalizados, con o sin régimen antibiótico (bajo la metodología de la Organización Mundial de la Salud) para determinar la prevalencia puntual sobre el uso de antibióticos. Resultados: Se identificaron 358 pacientes, la media de edad fue de 49 años y desviación estándar de 25,3 y fueron predominantemente adultos (88\%). El uso de antibióticos fue del 51,7\%; solo el 57,3\% de prescripciones siguió pautas basadas en guías de práctica clínica para indicación antibiótica, mientras que el $28,5 \%$ no seguían ninguna recomendación estandarizada. Las terapias fueron empíricas en el 86,8\% de pacientes y dirigidas en el 13,2\%. Conclusión: En más del 50\% de los pacientes hospitalizados se utilizaron antibióticos. Sin embargo, cerca de la tercera parte de prescripciones no seguían una indicación basada en alguna guía de práctica clínica. A pesar de existir un programa de control de antimicrobianos en el hospital, aún requiere optimizarse el uso adecuado de antimicrobianos.

Palabras clave: Antibióticos; Antiinfecciosos; Programas de Optimización del Uso de los Antimicrobianos (Fuente: DeCS BIREME).

\section{USE OF ANTIBIOTICS IN INPATIENTS FROM A NATIONAL HOSPITAL IN LIMA, PERU}

Citar como: Resurrección-Delgado C, Chiappe-Gonzalez A, BolarteEspinoza J, Martínez-Dionisio L, Muñante-Meneses R, VicenteLozano Y, et al. Uso de antibióticos en pacientes internados en un hospital nacional de Lima, Perú. Rev Peru Med Exp Salud Publica. 2020;37(4):620-6. doi: https://doi. org/10.17843/rpmesp.2020.374.5073

Correspondencia: Cristhian Pedro Resurrección Delgado; Calle De Las Letras N. ${ }^{\circ} 199$, Dpto. 1401, San Borja, Lima, Perú; cristhianprd27@hotmail.com

Recibido: $31 / 12 / 2019$

Aprobado: 29/07/2020

En línea: $04 / 11 / 2020$

\section{ABSTRACT}

Objective: To determine the point prevalence and characteristics of antibiotic use in different hospitalization areas of the Hospital Nacional Dos de Mayo. Materials and methods: We carried out a 3-week cross-sectional study with a census approach (using methodology from the World Health Organization) to determine the point prevalence of antibiotic use in inpatients from hospitalization areas, regardless of whether or not they were on an antibiotic regimen. Results: We identified 358 patients, with a mean age of $49( \pm 25.3)$ years, predominantly adults $(88 \%)$. Antibiotics were used in $51.7 \%$ of the hospitalized patients; only $57.3 \%$ of the prescriptions followed a regimen based on clinical practice guidelines for antibiotic treatment, while $28.5 \%$ did not follow any standardized recommendation. Therapies were empirical in $86.8 \%$ and directed in $13.2 \%$ of the cases. Conclusions: Antibiotics were used in more than $50 \%$ of hospitalized patients. However, about one third of the prescriptions were not based on any clinical practice guidelines. Despite the existence of an antimicrobial control program in the hospital, appropriate antimicrobial use still needs to be optimized.

Keywords: Antibiotics; Anti-Infective Agents; Antimicrobial Stewardship (Source: MeSH NLM).

\section{INTRODUCCIÓN}

El uso de antibióticos ha incrementado notoriamente la esperanza de vida de la población ${ }^{(1)}$. Sin embargo, la resistencia antibiótica (RA) es un problema cada vez más frecuente y uno de los grandes desafíos actuales de la salud pública ${ }^{(2)}$. El uso irracional o indiscriminado de 
antibióticos es la causa más importante de la resistencia antibiótica, y además trae consigo otras consecuencias adversas, como toxicidad, interacciones, infección por Clostridioides (Clostridium) difficile y estancia hospitalaria prolongada; lo cual finalmente incrementa la morbilidad, mortalidad y costos en el sistema de salud ${ }^{(3,4)}$.

Las tasas de RA son particularmente elevadas en los hospitales ${ }^{(5)}$. Se ha reconocido desde hace varias décadas que hasta el 50\% de los antibióticos prescritos tienen indicaciones innecesarias o inapropiadas ${ }^{(6)} \mathrm{y}$, adicionalmente, el escaso número de antibióticos recientemente aprobados reducen las opciones terapéuticas, lo que obliga al uso cada vez más prudente de los antibióticos. Por ello, con el propósito de abordar dicho problema la Organización Mundial de la Salud (OMS) promueve la instauración de Programas de Optimización de Antimicrobianos (PROA, por sus siglas en inglés). Los PROA son una estrategia efectiva que garantizan el uso correcto de los antibióticos, de acuerdo con la evidencia científica disponible, a fin de mejorar los resultados clínicos, minimizar los eventos adversos y reducir los costos hospitalarios ${ }^{(7)}$.

Existen datos sobre el uso de antimicrobianos en países de altos ingresos, donde las políticas de su empleo son más sólidas y donde se cuenta con mayores recursos y más experiencia en el desarrollo de los PROA ${ }^{(8)}$. Por otro lado, en países de medianos o de bajos ingresos, incluido el Perú, los PROA han venido implementándose en los últimos años, pese a la escasez de recursos humanos capacitados y las limitaciones económicas de los sistemas de salud ${ }^{(9,10)}$.

De esta manera, en el Perú se han iniciado acciones para frenar la resistencia a los antimicrobianos, a través de un Plan Multisectorial (2019-2021) ${ }^{(11)}$ y la elaboración de la Norma Técnica PROA. Sin embargo, aún contamos con limitada información acerca de la calidad y las características de la prescripción de antibióticos que nos sirvan de referencia para optimizar los programas de control de antimicrobianos. Por lo antedicho, presentamos un trabajo de prevalencia puntual en el uso de antibióticos, el cual brindará información de base sobre los patrones de prescripción de antibióticos en un hospital de tercer nivel en Lima, Perú.

\section{MATERIALES Y MÉTODOS}

\section{Población y muestra}

El Hospital Nacional Dos de Mayo (HNDM) es un hospital docente de referencia nacional, ubicado en Lima, Perú. Cuenta con 605 camas y con las principales especialidades médicas y quirúrgicas, excepto servicios de trasplantes y quemados. Las salas de hospitalización se dividen de la siguiente manera: seis salas de medicina, una sala de pediatría/cirugía pediátrica, cuatro salas de cirugía, una sala de traumatología y ortopedia, una sala de

\section{MENSAJES CLAVE}

Motivación para realizar el estudio: El uso racional de antibióticos es necesario para evitar la progresión de la resistencia a los antimicrobianos.

Principales hallazgos: Se realizó un estudio para evaluar el uso de antibióticos en un hospital de referencia nacional. La mitad de los pacientes recibió algún antibiótico y en 2 de cada 5 pacientes no había uso justificado de antibióticos. En 9 de cada 10 casos el tratamiento antibiótico fue empírico y no dirigido.

Implicancias: Es necesario reforzar a los médicos tratantes acerca de las recomendaciones para el adecuado uso racional de antibióticos basado en guías de práctica clínica y terapias dirigidas.

urología, una sala de obstetricia, una sala de neonatología, una sala de unidad de cuidados intensivos (UCI) pediátrica, tres salas de UCI adultos, una sala de UCI cardiovascular, una sala de infectología y medicina tropical, y una sala de neumología. Las salas de adultos de alto riesgo comprenden las de hematología y oncología médica. El término «otras salas» hace referencia a las áreas de cirugía pediátrica, neonatología, UCI neonatología y UCI adultos. Asimismo, se utilizaron las historias clínicas de pacientes hospitalizados (en el momento de la encuesta) de los departamentos de medicina, cirugía, ginecoobstetricia, pediatría y unidad de cuidados intensivos del HNDM.

La recolección de la información se realizó tomando los datos de la historia clínica y, en algunos casos cuando se necesitaba aclarar algún dato en particular, con una breve entrevista al médico tratante o enfermera. Asimismo, se obtuvo la información de todos los pacientes de una sala en el mismo día. Se incluyeron pacientes hospitalizados a las 8:00 a. m. del día del estudio, independientemente del uso o no de antibióticos. Se excluyeron los pacientes con indicación de alta, de consultorios externos, en sala de emergencia y hemodiálisis en el período de ejecución en la sala.

\section{Procedimiento}

\section{Instrumento}

Se realizó un estudio transversal de tres semanas de duración (del 3 al 21 de diciembre de 2018). El equipo de investigación conformado por médicos infectólogos y residentes de Infectología recibieron una capacitación sobre el llenado de los datos para la encuesta según la metodología de la OMS para la estimación de la prevalencia puntual sobre el uso de antibióticos en hospitales ${ }^{(12)}$. Se realizó un piloto previo para determinar el tiempo para recabar información, los errores en la consignación de información y las dudas en el llenado del cuestionario. Se recabó información de manera 
electrónica, y la mayoría de preguntas del cuestionario eran de opción múltiple.

El cuestionario de la encuesta fue validado y utilizado en estudios previos en idioma español ${ }^{(12)}$. Su estructura fue la siguiente: 1) información relacionada con el paciente (unidad de hospitalización, datos demográficos, fecha de ingreso y cirugías recientes durante la hospitalización), que se completó en todos los pacientes, tengan o no prescripción de antibióticos el día de la evaluación; 2) información sobre manejo de antibióticos (diagnóstico, microbiología, uso de antibióticos y la adherencia a guías clínicas). Se obtuvo la información de las historias clínicas, del kardex de enfermería, y del personal de salud de turno en las salas de hospitalización de medicina (médicos y enfermeras).

\section{Variables}

Las variables evaluadas fueron las siguientes: 1) Tipo de indicación para el uso de antibióticos, según el tipo de infección: adquirida en la comunidad (procedente de la comunidad) o adquirida 48 horas después de la hospitalización del paciente; 2) Motivo de la prescripción de antibióticos: tratamiento (evidencia o sospecha de infección), profilaxis (no evidencia de infección), desconocido; 3) Orientación del tratamiento: empírico (no evidencia de agente infeccioso o infección), dirigido (evidencia de agente infeccioso); 4) Dosis perdida: aquella que estando indicada por el médico, no fue administrada al paciente (verificada en el kardex de enfermería) y se consideraron omitidas por desabastecimiento o por otra causa (error en la administración, no tolerancia del paciente, desconocida); 5) Disponibilidad de resultados (cultivo con sensibilidad antimicrobiana) en el registro clínico; 6) Información de los resultados al médico tratante: el médico reporta que se le informaron los resultados; y 7) Acatamiento de sugerencias de Guías de Práctica Clínica (GPC).

La prescripción fue considerada adecuada cuando se ajustaba a una guía internacional o local, o contaba con el asesoramiento de un especialista en enfermedades infecciosas. Se consideró el no acatamiento cuando el médico tratante informaba que no utilizaba una GPC internacional o local para decidir la cobertura antibiótica empleada; o si tal decisión no era avalada por un médico especialista en enfermedades infecciosas mediante una evaluación o interconsulta.

\section{Análisis estadístico}

Previamente las variables se compilaron y analizaron en una base de datos en Microsoft Excel 2018. El análisis fue descriptivo, y se presentaron frecuencias absolutas y relativas (porcentuales) en tablas de frecuencias y gráficos de barras y circulares.

\section{Aspectos éticos}

Este estudio ha sido aprobado por el Comité de ética del HNDM (Reg. 014261). Dado el carácter observacional del estudio y la forma anónima en la que se recabaron los datos de las historias clínicas, el comité de ética decidió que no se requería el consentimiento informado de los pacientes.

\section{RESULTADOS}

Se identificaron 358 pacientes distribuidos en distintas salas de hospitalizacion del HNDM. La media de edad fue 49 [Desviación estándar (DE) de 25,3] años (<18 años: 25,7\%; 18-65 años: 53,9\%; >65 años: 34,1\%). La distribución por género fue similar (49,2\% varones; $50,5 \%$ mujeres y un paciente transgénero $0,3 \%)$. La mayoría de los pacientes fueron de las salas de medicina de adultos $(62,5 \%)$, cirugía adultos (17\%), neonatología (5\%), adultos de alto riesgo (4,7\%), medicina pediátrica $(4,2 \%)$ y de otras salas (adultos de alto riesgo $[3,3 \%]$, UCI pediátrica $[1,1 \%]$, gineco-obstetricia $[1,1 \%]$, cirugía pediátrica $[0,8 \%])$.

Los dispositivos invasivos estuvieron presentes en 341 casos $(95,2 \%)$. El más frecuente fue el catéter venoso periférico $(90,5 \%)$, seguido del vesical $(16,5 \%)$ y del venoso central (10,9\%), mientras que los catéteres de hemodiálisis, peritoneal y otros representaron el 7\%. Se realizó intubación orotraqueal en el $8,4 \%$ de pacientes, y se realizó algún procedimiento quirúrgico en el $23,5 \%$ de los pacientes durante su hospitalización.

Se usó antibióticos en el 51,7\% de pacientes; con más frecuencia en salas de pediatría $(73,3 \%)$, cirugía pediátrica $(66,7 \%)$, medicina adultos $(57,1 \%)$, neonatología (50\%), gineco-obstetricia (50\%) y cirugía de adultos (42,6\%); y con menos frecuencia en UCI adultos (29,4\%), UCI pediátrica (25\%) y hospitalización adultos de alto riesgo (8,3\%) (Tabla 1).

El motivo principal para usar antibióticos fueron las infecciones adquiridas en la comunidad $(66,1 \%)$, seguida por las relacionadas al cuidado de la salud (19\%). Los diagnósticos más frecuentes para el uso de antibióticos fueron neumonía (19,6\%), infección a la piel y partes blandas sin osteomielitis no relacionada con cirugía $(12,1 \%)$, infección urinaria baja $(11,1 \%)$, infección intraabdominal $(8,9 \%)$, sepsis clínica $(7,4 \%)$, infección urinaria alta $(4,2 \%)$. Los diagnósticos menos frecuentes fueron bronquitis aguda o crónica exacerba$\mathrm{da}$, bacteriuria asintomática y síndrome inflamatorio de respuesta sistémica que recibieron antibióticos en el 2,6\%, 2,1\% y $0,5 \%$ de los casos; respectivamente (Tabla 1).

Se indicó el uso de antibióticos como tratamiento $(87,8 \%)$, por profilaxis quirúrgica $(7,9 \%)$ y por motivos desconocidos $(4,2 \%)$. Todas las indicaciones de profilaxis quirúrgica recibieron dosis múltiples del fármaco en un mismo día (Tabla 1).

Referente al número de antibióticos empleados por paciente, 49,7\% recibieron un antibiótico; 44,9\% dos antibióticos y 5,4\%, tres o más antibióticos. Solo el 57,3\% de las prescripciones en pacientes siguió pautas basadas en GPC locales o internacionales para indicación antibiótica, mien- 
Tabla 1. Prevalencia puntual en el uso de antibióticos $(\mathrm{n}=358)$

\begin{tabular}{lr}
\hline Características & \multicolumn{1}{c}{ n (\%) } \\
\hline Prevalencia global & $185(51,7)$ \\
Distribución por salas & \\
Hospitalización medicina pediátrica & $11(73,3)$ \\
Hospitalización cirugía pediátrica & $2(66,7)$ \\
Hospitalización medicina adultos & $128(57,1)$ \\
Hospitalización neonatología & $9(50,0)$ \\
Hospitalización gineco-obstetricia & $2(50,0)$ \\
Hospitalización cirugía adultos & $26(42,6)$ \\
UCI adultos & $5(29,4)$ \\
UCI pediátrica & $1(25,0)$ \\
Sala de adultos de alto riesgo & $1(8,3)$ \\
Tipo de indicación & \\
IRCS & $36(19,0)$ \\
Infecciones comunitarias & $125(66,1)$ \\
Desconocido & $7(3,7)$ \\
Otro & $21(11,1)$ \\
Propósito de la indicación & \\
Profilaxis quirúrgica & $15(7,9)$ \\
Tratamiento & $166(87,8)$ \\
Desconocido & $8(4,2)$ \\
Duración de la profilaxis quirúrgica & \\
Monodosis & \\
Dosis múltiples en un día & 0 \\
Dosis múltiples en más de un día & $15(100)$ \\
Desconocido & 0 \\
\hline
\end{tabular}

UCI: unidad de cuidados intensivos; IRCS: infecciones relacionadas a cuidados de salud.

tras que el $28,5 \%$ no seguía ninguna recomendación estandarizada. La frecuencia en salas hospitalarias sobre el uso de antibióticos acorde a GPC locales o internacionales fue adultos de alto riesgo (100\%), neonatología (94,4\%), medicina adultos $(77,7 \%)$, medicina pediátrica $(71,4 \%)$, cirugía pe- diátrica $(66,7 \%)$, cirugía adultos $(48,7 \%)$, gineco-obstetricia (25\%) y UCI adultos (20\%) (Figura 1).

En $141(49,8 \%)$ casos, el prescriptor inicial de antibióticos fue el médico especialista, mientras que en el 50,2\% restante fue el médico residente. El 86,8\% de las terapias fueron empíricas. Asimismo, el 37\% de los pacientes tenía alguna muestra biológica recolectada para cultivo microbiológico (sangre [22,7\%], orina [43,3\%], esputo [11,3\%], secreción de herida [6,3\%], fluidos estériles [7,5\%] y otros [8,8\%]). Finalmente, en el $71 \%$ de los casos, se notificó al médico tratante los resultados de cultivos (Tabla 2).

En $64(22,7 \%)$ pacientes, se perdió al menos una dosis antibiótica requerida, con una mediana de dosis perdida de 2,9 (rango intercuartílico: 1-16) y por consecuencia del desabastecimiento en 48,5\% de los casos. Meropenem, vancomicina y ceftriaxona fueron los antibióticos empleados con mayor frecuencia para infecciones comunitarias e infecciones relacionadas a cuidados de la salud (Tabla 3 ).

\section{DISCUSIÓN}

La frecuencia general del uso de antibióticos encontrada $(51,7 \%)$ fue similar al de otros estudios de prevalencia puntual $^{(13-15)}$. Estudios en la región de las Américas, reflejan datos similares al de nuestra realidad. Por ejemplo, en 2011, en hospitales de cuidados agudos de diez estados de EE. UU. se describió que cerca del 50\% de los pacientes incluidos en el estudio, recibieron algún antibiótico ${ }^{(13)}$. En 2015, otro estudio que incluyó cuatro países de Latinoamérica (Argentina, Brasil, Chile y México) describió que en el 47,5\% de los pacientes reclutados usaron antibióticos, de los cuales, el $90 \%$ eran de uso sistémico ${ }^{(14)}$. En otro estudio de prevalencia puntual de uso de antibióticos realizado en población mexicana entre agosto y setiembre de 2016, se describió que

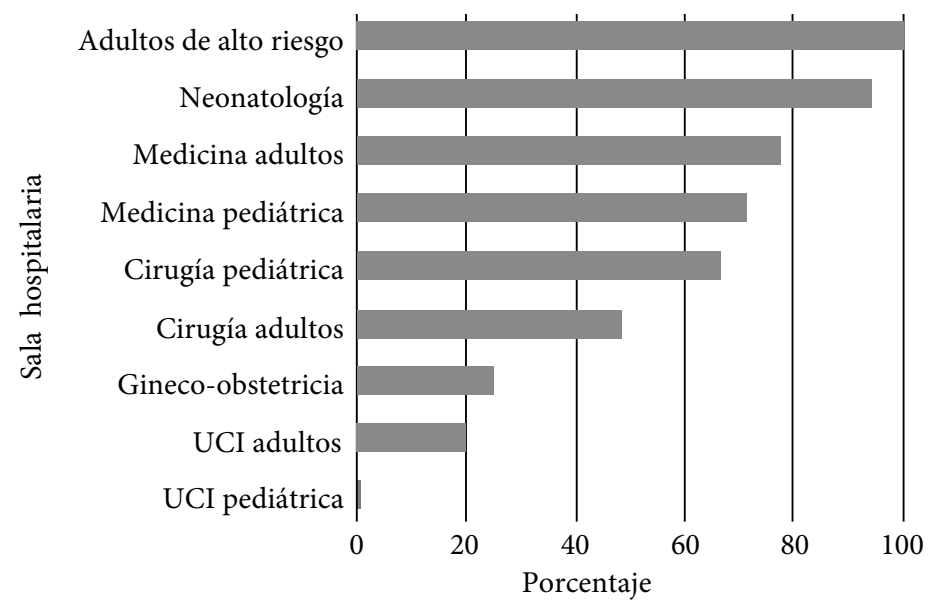

UCI: unidad de cuidados intensivos

Figura 1. Acatamiento de las sugerencias de guías de práctica clínica locales o internacionales según sala hospitalaria en el Hospital Nacional Dos de Mayo, 2018. 
Tabla 2. Características del uso de antibióticos en el Hospital Nacional Dos de Mayo, 2018 ( $\mathrm{n}=358)$.

\begin{tabular}{|c|c|}
\hline Características & n (\%) \\
\hline \multicolumn{2}{|l|}{ Tipo de prescriptor } \\
\hline Especialista & $141(49,9)$ \\
\hline Médico residente & $142(50,1)$ \\
\hline \multicolumn{2}{|l|}{ Tipo de orientación para el tratamiento } \\
\hline Empírico & $145(86,8)$ \\
\hline Dirigido & $22(13,2)$ \\
\hline Desconocido & 0 \\
\hline \multicolumn{2}{|l|}{ Alguna dosis antibiótica perdida } \\
\hline Sí & $64(22,7)$ \\
\hline No & $202(71,6)$ \\
\hline Desconocido & $16(5,6)$ \\
\hline Promedio de dosis perdidas (media, RIC) & $2,9(1-16)$ \\
\hline \multicolumn{2}{|l|}{ Motivos de pérdida de las dosis } \\
\hline $\begin{array}{l}\text { Todas las dosis omitidas por } \\
\text { desabastecimiento }\end{array}$ & $4(6,3)$ \\
\hline $\begin{array}{l}\text { Ninguna dosis omitida por } \\
\text { desabastecimiento }\end{array}$ & $1(1,6)$ \\
\hline $\begin{array}{l}\text { Algunas dosis omitidas por } \\
\text { desabastecimiento }\end{array}$ & $27(42,2)$ \\
\hline Desconocida & $32(50,0)$ \\
\hline \multicolumn{2}{|l|}{$\begin{array}{l}\text { Toma de muestra para diagnóstico } \\
\text { microbiológico }\end{array}$} \\
\hline Sí & $70(37,0)$ \\
\hline No & $86(45,5)$ \\
\hline Desconocido & $33(17,5)$ \\
\hline \multicolumn{2}{|l|}{ Tipo de muestra } \\
\hline Sangre & $18(22,7)$ \\
\hline Orina & $34(43,3)$ \\
\hline Esputo & $9(11,3)$ \\
\hline Secreción de herida & $5(6,3)$ \\
\hline Fluidos estériles & $6(7,5)$ \\
\hline Otro & $7(8,8)$ \\
\hline Disponibilidad de resultados de cultivo & $51(72,9)$ \\
\hline $\begin{array}{l}\text { Disponibilidad de pruebas de sensibilidad } \\
\text { antimicrobiana }\end{array}$ & $37(53,3)$ \\
\hline $\begin{array}{l}\text { Información de resultados a médico } \\
\text { tratante }\end{array}$ & $49(71,0)$ \\
\hline
\end{tabular}

RIC: rango intercuartil.

de 260 pacientes incluidos el 51\% recibió al menos un antibiótico ${ }^{(15)}$.

Por otro lado, la realidad europea presenta algunos contrastes con respecto a la nuestra. En 2016, un estudio del centro nacional de vigilancia de infecciones nosocomiales de Alemania describió que de 218 hospitales y 64412 pacientes observados, la prevalencia de uso de antibióticos fue del $26 \%{ }^{(16)}$. Adicionalmente, en hospitales de cuidados agudos de Europa se evidenció que la tercera parte de los pacientes incluidos recibieron al menos un antibiótico (70\% un antibiótico, $24 \%$ dos antibióticos, $4,5 \%$ tres, y $1,3 \%$ cuatro o más antibióticos) en $2017^{(17)}$. Esto refuerza el concepto que en la última década, la frecuencia de uso de antibióticos por prevalencia puntual es menor en regiones de Europa ${ }^{(16,17)}$ que en unidades de cuidados críticos de América ${ }^{(13-15)}$.

El 66\% de los pacientes recibieron terapia antibiótica por infecciones adquiridas en la comunidad, similar a otros estudios que reportan 76,5\% (México, 2016) ${ }^{(15)} ; 70 \%$ (EE. UU., 2011) ${ }^{(13) ;}$ y $60,5 \%$ (Latinoamérica, 2015) ${ }^{(14)}$. Mientras que en Europa es menor $(45 \%)^{(17)}$. Los estudios mencionados, incluso el nuestro, coinciden en que el diagnóstico más frecuente para la prescripción antimicrobiana fueron las infecciones respiratorias bajas.

Los trabajos citados muestran que los antibióticos utilizados con más frecuencia en infecciones adquiridas en la comunidad fueron las penicilinas con inhibidores de betalactamasas, seguidas de las cefalosporinas y las fluoroquinolonas ${ }^{(16,17,21-23)}$, conductas relacionadas con el uso racional de antibióticos y menor estancia hospitalaria ${ }^{(18)}$. En algunas series como la nuestra, predominantemente de países latinoamericanos ${ }^{(14)}$, se describe también el uso de antibióticos de amplio espectro en contextos que no necesariamente lo ameritaban, como en la hospitalización fuera de la unidad de cuidados críticos ${ }^{(19)} \mathrm{e}$ infecciones adquiridas en la comunidad ${ }^{(13,15)}$.

A pesar de que en el $88 \%$ de los pacientes que utilizaron antibióticos (por tratamiento de una patología infecciosa), cerca de la tercera parte de estos no seguían una indicación basada en alguna GPC. Hallazgos similares se muestran en diversos estudios estadounidenses, como el del hospital universitario de Ohio, donde el 30\% de las indicaciones de antibióticos fueron considerados como innecesarias ${ }^{(20)}$. En México (2016) ${ }^{(15)}$, el 21\% de los pacientes con uso de antibiótico no tuvo justificación de emplearse. De igual manera, en Latinoamérica (Argentina, Brasil, Chile, México) solo llegaba al 64\%. En contraste, otras realidades como en hospitales de Australia (2014) ${ }^{(21)}$, el porcentaje de prescripciones inadecuadas fue cerca de la cuarta parte y el uso no direccionado por guías fue del $27 \%$. Otro estudio multicéntrico $(2015)^{(14)}$ reportó un porcentaje similar $(77,4 \%)$ de adherencia a GPC, y finalmente un estudio en Singapur ${ }^{(23)}$ muestra que fue infrecuente el uso de antibiótico sin justificación $(0,4 \%)$. Las causas descritas de uso no justificado de antibióticos fueron los tiempos prolongados de terapia, los procesos no infecciosos, y los tratamientos de hallazgos de microorganismos colonizantes ${ }^{(20)}$.

La profilaxis antibiótica en cirugía representó el 8\% de la totalidad de las indicaciones en nuestro estudio, resaltando el uso irracional de la misma, al exceder el día de administración de antibiótico. De esta manera, se señala que se prescribieron múltiples dosis y con una duración superior a las 24 horas en casi la totalidad de las indicaciones. En otras realidades, como Australia, este comportamiento se observa en el $40 \%{ }^{(21)}$ y en países 
Tabla 3. Distribución antibiótica por tipo de indicación en el Hospital Nacional Dos de Mayo, 2018 (n = 189).

\begin{tabular}{|c|c|c|c|c|}
\hline Antibiótico & $\begin{array}{c}\text { IRCS } \\
(\mathrm{n}=36)\end{array}$ & $\begin{array}{c}\text { IC } \\
(n=125)\end{array}$ & $\begin{array}{c}\text { Desconocido } \\
(\mathbf{n}=7)\end{array}$ & $\begin{array}{c}\text { Otro } \\
(\mathbf{n}=21)\end{array}$ \\
\hline Amikacina & 1 & 3 & $3^{\mathrm{h}}$ & 5 \\
\hline Azitromicina & 0 & $4^{\mathrm{e}}$ & 0 & 0 \\
\hline Ceftazidima & 5 & $12^{\mathrm{f}, \mathrm{g}}$ & $2^{\mathrm{h}}$ & 1 \\
\hline Ceftriaxona & 9 & $49^{\mathrm{d}, \mathrm{e}}$ & $2^{\mathrm{d}}$ & 3 \\
\hline Ciprofloxacino & $3^{\mathrm{a}}$ & $13^{\mathrm{a}, \mathrm{g}}$ & $1^{\mathrm{a}}$ & 1 \\
\hline Clindamicina & $2^{\mathrm{a}}$ & $17^{\mathrm{a}, \mathrm{d}, \mathrm{f}}$ & $2^{\mathrm{a}, \mathrm{d}}$ & 3 \\
\hline Colistina & $2^{\mathrm{b}}$ & 0 & 0 & 0 \\
\hline Ertapenem & 2 & 0 & 0 & 0 \\
\hline Imipenem & $3^{c}$ & 4 & 0 & 0 \\
\hline Meropenem & $8^{\mathrm{b}, \mathrm{c}}$ & $27^{\mathrm{c}}$ & 0 & 3 \\
\hline
\end{tabular}

El número de antibióticos es mayor al número total de cada tipo de indicación debido a la antibioticoterapia combinada.

IRCS: infecciones relacionadas a cuidados en salud; IC: infecciones comunitaria.

${ }^{\text {a }}$ ciprofloxacino + clindamicina (IRCS: 1; IC: 4; desconocido: 1); ${ }^{\text {b }}$ colistina + meropenem (IRCS: 2 ); ${ }^{\mathrm{c}}$ carbapenem +vancomicina (IRCS: 4 ; IC: 5 ); ${ }^{\mathrm{d}}$ ceftriaxona + clindamicina (IC: 3 ; desconocido: 1 ); ${ }^{\mathrm{e}}$ ceftriaxona + azitromicina (IC: 3 ); ${ }^{\mathrm{f}}$ ceftazidima + clindamicina (IC: 2 ); ${ }^{\mathrm{g}}$ ceftazidima + ciprofloxacino (IC: 5 ); ${ }^{\mathrm{h}}$ ceftazidima + amikacina (desconocido: 2).

de Europa entre el 54\% y 56\% ${ }^{(16,17)}$. En contraposición, un estudio llevado a cabo en 13 hospitales de Singapur evidenció que solo el $8,4 \%$ de las prescripciones en profilaxis quirúrgica tuvieron una duración superior a un día ${ }^{(23)}$. Estos datos revelan que existe mucho por trabajar en la concientización hacia los profesionales de especialidades quirúrgicas en la duración de la profilaxis antibiótica, puesto que ello trae como consecuencia el incremento de la resistencia antibiótica.

Con relación al aislamiento microbiológico, nuestro estudio resalta que únicamente en el $37 \%$ de los casos se tomó alguna muestra microbiológica para hallar el posible agente causal del cuadro clínico, y a su vez, la mayoría de dichas muestras $(77,3 \%)$ consistían en sangre, orina y esputo. En un estudio retrospectivo llevado a cabo en el centro médico universitario de la Universidad de Ámsterdam, Países Bajos, con 733 camas de internamiento, se realizan alrededor de 7500 hemocultivos anualmente, contrastando la cultura institucional de cultivar con nuestras cifras ${ }^{(24)}$. En el estudio de hospitales de Singapur (2015-2016) ${ }^{(23)}$, el 45,5\% presentó algún cultivo microbiológico positivo.

Debido al limitado número de camas, la UCI pediátrica $\mathrm{y}$ la de adultos representaron un pequeño porcentaje $(1,1 \% \mathrm{y}$ $4,7 \%$, respectivamente) del total de pacientes evaluados. No obstante, se evidenció un menor porcentaje en el uso de antibióticos en dichas unidades (25\% en UCI pediátrica y 29,4\% en UCI de adultos), lo que sugiere una mayor intervención del PROA del HNDM en las salas mencionadas. El último consenso promovido por la Joint Programming Initiative on Antimicrobial Resistance (2019) estandariza sugerencias para el óptimo seguimiento y monitoreo de los programas de control de antimicrobianos, y hace hincapié en el ítem referente a la medición del uso de drogas, donde se estipula que la dosis diaria definida (DDD, por sus siglas en inglés) o días de terapia (DOT, por sus siglas en inglés) son los mejores parámetros para su evaluación ${ }^{(25)}$.

Los antibióticos usados con más frecuencia fueron ceftriaxona $(22,2 \%)$, meropenem $(12,8 \%)$, vancomicina $(10,8 \%)$, clindamicina (8\%), ceftazidima $(6,7 \%)$ y ciprofloxacino (6\%). Se remarcan los porcentajes muy similares en el empleo de meropenem y vancomicina tanto para infecciones nosocomiales y comunitarias, al igual que el porcentaje no desdeñable del uso de ceftriaxona para infecciones nosocomiales. Lo antedicho refleja el empleo no prudente de antibióticos en nuestra realidad.

El presente estudio no evaluó el consumo de antibióticos en DDD, el desenlace del paciente y la duración de la terapia antibiótica, debido al diseño del estudio transversal que buscó describir en un momento determinado las características del uso de antibióticos en pacientes hospitalizados. Por ello, estas variables podrían considerarse en próximos estudios.

En conclusión, este estudio describe una elevada frecuencia de uso irracional de antibióticos en pacientes hospitalizados, con frecuencias de $15-25 \%$ de uso no justificado de cefalosporinas, clindamicina y amikacina, además del $13 \%$ de uso de carbapenémicos en infecciones comunitarias. En cerca del $90 \%$ de pacientes que recibieron antibióticos, la prescripción fue empírica y no dirigida. En nuestro hospital, se requieren medidas de acción urgentes a través de la solidificación y potenciación del PROA institucional, para reflejar menores tasas de uso innecesario e inadecuado de antibióticos, empleo acorde a GPC, acceso oportuno a los antibióticos y menores cifras de resistencia bacteriana. 
Contribuciones de los autores: PRG, WCV, CRD, ACG, JMI, RMM, YVL y LMD recolectaron la información por diversos pabellones de internamiento del Hospital Nacional Dos de Mayo. CRD, ACG, JMI, JAB y JBE diseñaron el manuscrito y su redacción. CRD, ACG, JAB, JBE y JMI analizaron los resultados. Todos los autores hicieron la revisión crítica del artículo por separado y posteriormente unificaron conceptos. PRG, WCV, CRD, ACG, JMI, RMM,

\section{REFERENCIAS BIBLIOGRÁFICAS}

1. Hollis A, Ahmed Z. Preserving Antibiotic: Rationally. N Engl J Med. 2013;369(26):2474-6. doi: 10.1056/NEJMp1311479.

2. World Health Organization. Antimicrobial resistance: global report on surveillance [Internet]. Geneva, Switzerland; 2014 [citado el 27 de mayo de 2020]. Disponible en: http://www.who.int/iris/handle/10665/112642.

3. Shaughnessy MK, Amundson WH, Kuskowski MA, DeCarolis DD, Johnson JR, Drekonja DM. Unnecessary antimicrobial use in patients with current or recent Clostridium difficile infection. Infect Control Hosp Epidemiol. 2013;34(2):109-16. doi: 10.1086/669089.

4. Beardsley JR, Williamson JC, Johnson JW, Luther VP, Wrenn RH, Ohl CC. Show me the money: long-term financial impact of an antimicrobial stewardship program. Infect Control Hosp Epidemiol. 2012;33(4):398400. doi: $10.1086 / 664922$

5. Nasr P. Genetics, Epidemiology and Clinical Manifestations of Multidrug-Resistant Acinetobacter baumannii. J Hosp Infect. 2020;104(1):4-11. doi: 10.1016/j.jhin.2019.09.021.

6. Centers for Disease Control and Prevention, Office of Infectious Disease [Internet]. Antibiotic resistance threats in the United States, 2013; 2013. [citado el 27 de mayo de 2020]. Disponible en: https://www.cdc.gov/ drugresistance/pdf/ar-threats-2013-508.pdf.

7. Cisneros J, Pérez-Moreno M, Gil-Navarro M. Política de antibióticos. Comisión de infecciones y uso de antimicrobianos. Enferm Infecc Microbiol Clin. 2014;32(8):533-6. doi: 10.1016/j.eimc.2014.01.008.

8. Barlam T, Cosgrove S, Abbo L, MacDougall C, Schuetz A, Septimus E, et al. Implementing an Antibiotic Stewardship Program: Guidelines by the Infectious Diseases Society of America and the Society for Healthcare Epidemiology of America. Clin Infect Dis. 2016;62(10):e51-e77. doi: 10.1093/cid/ciw118.

9. World Health Organization. Antimicrobial stewardship programmes in health-care facilities in low- and middle-income countries: a WHO practical toolkit [Internet]. WHO; 2019 [citado el 27 de mayo de 2020]. Disponible en: https://apps.who.int/iris/handle/10665/329404.

10. Van Dijck C, Vlieghe E, Cox J. Antibiotic stewardship interventions in hospitals in low-and middle income countries: a systematic review. Bull World Health Organ. 2018;96(4):266-80. doi: 10.2471/BLT.17.203448.

11. Ministerio de Salud. DS No010-2019-SA Aprueban el "Plan Multisectorial para enfrentar la Resistencia a los Antimicrobianos 2019-2021" que como Anexo forma parte integrante del presente Decreto Supremo [Internet]. MINSA; 2019 [citado el 27 de mayo de 2020]. Disponible en: https://www. gob.pe/institucion/minsa/normas-legales/276868-010-2019-sa.

12. Global PPS Development Group. Point Prevalence Survey of Antimicrobial Consumption and Resistance [Internet]. GLOBAL-PPS; 2017 [citado el 27 de mayo de 2020]. Disponible en: http://www.global-pps. com/wp-content/uploads/GLOBAL-PPS-2017-Protocol.pdf.

13. Magill SS, Edwards JR, Beldavs ZG, Dumyati G, Janelle SJ, Kainer MA, et al. Prevalence of Antimicrobial Use in US Acute Care Hospitals, May-September 2011. JAMA. 2014;312(14):1438-46. doi: 10.1001/ jama.2014.12923.

14. Versporten A, Zarb P, Caniaux I, Gros MF, Drapier N, Miller M, et al. Global-PPS network. Antimicrobial consumption and resistance in adult hospital inpatients in 53 countries: results of an internet-based global
YVL y LMD realizaron las correcciones del artículo. Todos los autores asumen responsabilidad frente a todos los aspectos del manuscrito y realizaron la aprobación de la versión final.

Financiamiento: Autofinanciado.

Conflictos de interés: Los autores declaran no tener ningún conflicto de interés.

point prevalence survey. Lancet Glob Health. 2018;6(6):e619-e629. doi 10.1016/S2214-109X(18)30186-4.

15. Soria-Orozco M, Padrón-Salas A, González-Mercado JJ, Villava-von der Heyde N, Valerdi-Contreras L, López-Iñiguez Á, et al. Prevalence of antimicrobial use among hospitalized patients in non-critical areas in a university hospital in Mexico. Salud Publica Mex. 2017;59(5):504-505. doi: $10.21149 / 8465$.

16. Aghdassi SJS, Gastmeier P, Piening BC, Behnke M, Peña Diaz LA, Gropmann A, et al. Antimicrobial usage in German acute care hospitals: results of the third national point prevalence survey and comparison with previous national point prevalence surveys. J Antimicrob Chemother. 2018;73(4):1077-1083. doi: 10.1093/jac/dkx494.

17. Plachouras D, Kärki T, Hansen S, Hopkins S, Lyytikäinen O, Moro ML, et al. The Point Prevalence Survey Study Group. Antimicrobial use in European acute care hospitals: results from the second point prevalence survey (PPS) of healthcare-associated infections and antimicrobial use, 2016 to 2017. Euro Surveill. 2018;23(46):1800393. doi: 10.2807/1560-7917. ES.23.46.1800393.

18. Ufer M, Radosević N, Vogt A, Palcevski G, Francetić I, Reinalter SC, et al. Antimicrobial drug use in hospitalised paediatric patients: a cross-national comparison between Germany and Croatia. Pharmacoepidemiol Drug Saf. 2005;14(10):735-9. doi: 10.1002/pds.1108.

19. Stenehjem E, Hersh AL, Sheng X, Jones P, Buckel WR, Lloyd JF, et al. Antibiotic Use in Small Community Hospitals. Clin Infect Dis. 2016;63(10):1273-1280. doi: 10.1093/cid/ciw588.

20. Hecker MT, Aron DC, Patel NP, Lehmann MK, Donskey CJ. Unnecessary use of antimicrobials in hospitalized patients: current patterns of misuse with an emphasis on the antianaerobic spectrum of activity. Arch Intern Med. 2003;163(8):972-8. doi: 10.1001/archinte.163.8.972.

21. Turnidge JD, Thursky K, Chen CS, McNeil VR, Wilkinson IJ. Antimicrobial use in Australian hospitals: how much and how appropriate?. Med J Aust. 2016;205(10):S16-S20. doi: 10.5694/mja15.00899.

22. Kuster SP, RuefC, Ledergerber B, Hintermann A, Deplazes C, Neuber L, et al. Quantitative antibiotic use in hospitals: comparison of measurements, literature review, and recommendations for a standard of reporting. Infection. 2008;36(6):549-59. doi: 10.1007/s15010-008-7462-z.

23. Cai Y, Venkatachalam I, Tee NW, Tan TY, Kurup A, Wong SY, et al. Prevalence of Healthcare-Associated Infections and Antimicrobial Use Among Adult Inpatients in Singapore Acute-Care Hospitals: Results From the First National Point Prevalence Survey. Clin Infect Dis. 2017;64(2):S61-S67. doi: 10.1093/cid/cix103.

24. Nannan Panday RS, Wang S, Van de Ven PM, Hekker TAM, Alam N, Nanayakkara PWB. Evaluation of blood culture epidemiology and efficiency in a large European teaching hospital. PLoS One. 2019;14(3):e0214052. doi: 10.1371/journal.pone.0214052.

25. Schweitzer VA, Van Werkhoven $\mathrm{CH}$, Rodríguez Baño J, Bielicki J, Harbarth S, Hulscher M, et al. Optimizing design of research to evaluate antibiotic stewardship interventions: consensus recommendations of a multinational working group. Clin Microbiol Infect. 2019;26(1):41-50. doi: 10.1016/j.cmi.2019.08.017. 